Int. J. Dev. Biol. 61: 465-470 (2017)

doi: $10.1387 / \mathrm{ijdb} .170037 \mathrm{af}$

\title{
Expression of the ALK1 family of type I BMP/ADMP receptors during gastrula stages in Xenopus embryos
}

\author{
AVI LEIBOVICH ${ }^{1}$, HERBERT STEINBEIßER ${ }^{2}$ and ABRAHAM FAINSOD*,1 \\ ${ }^{1}$ Department of Developmental Biology and Cancer Research, Institute for Medical Research Israel-Canada, Faculty of Medicine, \\ Hebrew University, Jerusalem, Israel and ${ }^{2}$ Institute of Human Genetics, University Hospital Heidelberg, Heidelberg, Germany
}

\begin{abstract}
Multiple members of the transforming growth factor beta (TGF $\beta$ ) family of secreted factors play central inductive and patterning roles during embryogenesis. During gastrulation in vertebrates, the bone morphogenetic protein (BMP) sub-family is linked to formation of the embryonic organizer, Spemann's organizer in Xenopus, and dorsal-ventral mesoderm patterning. Our knowledge regarding the BMP receptors mediating this signaling is still very incomplete. The BMPR1A (ALK3) and BMPR1B (ALK6) receptors are known to mediate the BMP4 signal. These receptors belong to the ALK1 subfamily of type I receptors that also includes ACVR1 (ALK2), and ACVRL1 (ALK1). We studied by qPCR and in situ hybridization the spatio-temporal expression patterns of ALK2 and ALK1 and compared them to ALK3 and ALK6, and to the main BMPs expressed during gastrulation, i.e., BMP4, BMP7, BMP2, and ADMP, in an attempt to establish a link between ligands and receptors. There is extensive overlap between BMP4, and ALk3 and Alk6 expression, supporting their functional interaction. Robust $A / k 6$ expression was observed from mid-gastrula. Animal region expression of both receptors shows co-expression with BMP4 and BMP7. Alk2 transcripts were detected within the organizer, overlapping with its proposed ligand, $A D M P$, suggesting a probable function within the organizer. Alk1 is very weakly expressed during gastrula, but its transcripts were localized to the lateral marginal zone flanking the organizer domain. No receptor closely matched the maternal BMP2 expression, although Alk2, Alk3, and Alk6, have transcripts of maternal origin. Our analysis shows that the BMP ligands and their receptors exhibit dynamic expression patterns during gastrula stages.
\end{abstract}

KEY WORDS: TGF $\beta$ receptor, ALK receptor, BMP signaling, Xenopus embryo, anti-dorsalizing morphogenetic protein

BMP signaling is a complex signaling network involving numerous ligands and receptors. The ligands function as homo- or heterodimeric proteins, while the receptors are hetero-tetrameric complexes comprised of two type I and two type II receptors (Heldin and Moustakas 2016). The type I family of TGF $\beta$ receptors, also known as activin receptor-like kinases (ALKs) has seven members while the type II family has five. Therefore, BMP signaling can exhibit a high level of complexity as a result of the multiple ligand possibilities and receptor combinations. This pathway is further regulated extracellularly by secreted antagonists, decoys, and proteases cleaving the precursor ligand proteins or the secreted antagonists. Intracellularly, the BMP signaling pathway mainly mediates its effects through phosphorylation of the Smad proteins, but alternative signaling possibilities also take place (Heldin and
Moustakas 2016). This complexity allows for precise patterning of embryonic tissues by establishing morphogen gradients and regulating gene expression through dose-dependent responses (Dosch et al., 1997; Marom et al., 1999).

In Xenopus embryos BMP signaling plays a central role in the formation of Spemann's organizer, the embryonic organizer, and during dorsal-ventral patterning of the mesoderm (Dosch et al., 1997; Marom et al., 1999; Marom et al., 2005). The important roles of BMP signaling during early embryogenesis prompted the

Abbreviations used in this paper: ADMP, anti-dorsalizing morphogenetic protein; ALK, activin receptor-like kinase; BMP, bone morphogenetic protein; ISH , in situ hybridization; qPCR, quantitative real-time reverse transcription PCR; TGF, transforming growth factor.

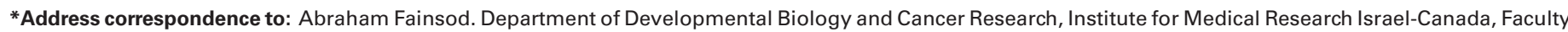
of Medicine, Hebrew University, Jerusalem 9112102, Israel. Tel: +972-2-6758157. Fax: +972-2-6757482. E-mail: abrahamf@ekmd.huji.ac.il

(iD) http://orcid.org/0000-0002-6368-0416
}

Supplementary Material (1 figure) for this paper is available at: http://dx.doi.org/10.1387/ijdb.170037af

Submitted: 20 February, 2017; Accepted: 8 May, 2017.

ISSN: Online 1696-3547, Print 0214-6282

(c) 2017 UPV/EHU Press

Printed in Spain 


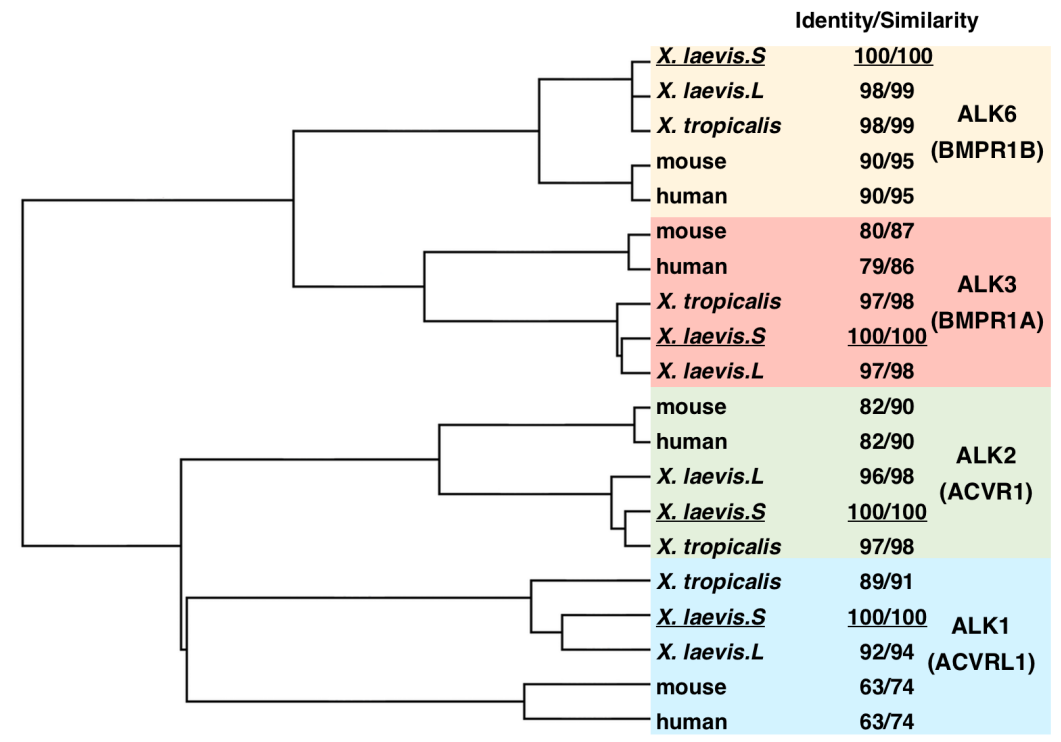

Fig. 1. The ALK1 family of type I bone morphogenetic protein (BMP) receptors. Phylogenetic tree of the ALK1family of type I BMP receptors. Protein sequences from Xenopus laevis and Xenopus tropicalis, human and mouse, were included in the analysis. The analysis subdivided the ALK1 sub-families, ALK6, ALK3, ALK2, and ALK1. For each sub-family, the percent sequence identity to the relevant Xenopus laevis protein is shown. characterization of multiple components and interactions of this signaling pathway in frog embryos. While BMP2 transcripts are present as a strong maternal contribution (Marom et al., 2005), BMP7, BMP4 and ADMP are expressed mainly from zygotic transcription (Fainsod et al., 1994; Moos et al., 1995; Hawley et al., 1995).

Our knowledge of the expression of the BMP receptors and their ligand specificity remains fragmentary. Here we describe the temporal and spatial pattern of expression of the ACVR1 (ALK2) and ACVRL1 (ALK1) type I receptors. We compare their expression patterns during gastrula stages to the well-studied BMP4 type I receptors BMPR1A (ALK3) and BMPR1B (ALK6) (Graff et al., 1994; Schille et al., 2016) and their putative ligands, BMP4, BMP2, BMP7, and ADMP. Our results show a very complex set of ligand and receptor expression patterns contributing to the regulated establishment of the organizer domain and subsequently the BMP morphogen gradient.

\section{Results and Discussion}

The BMP family of signaling factors binds mainly
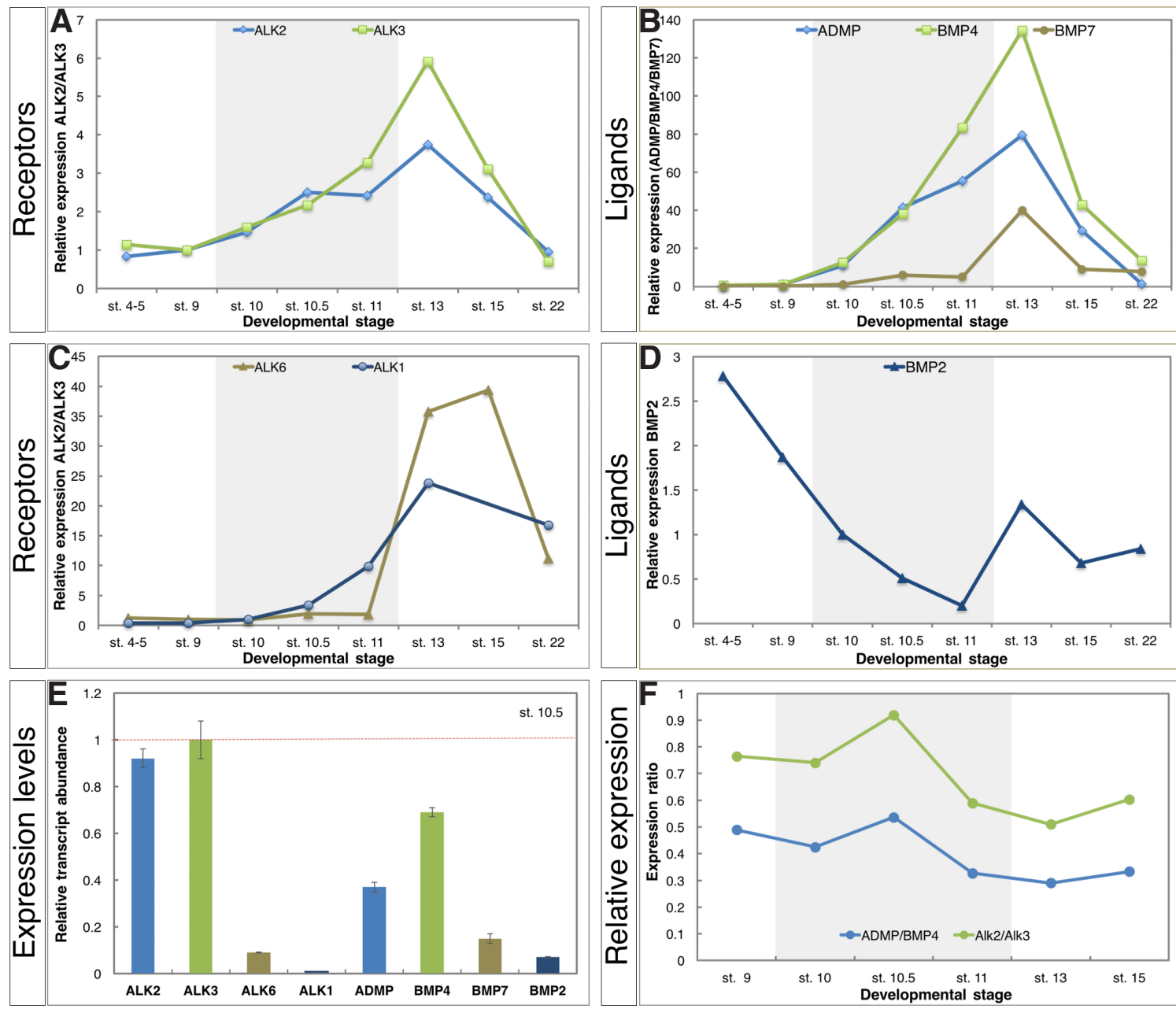

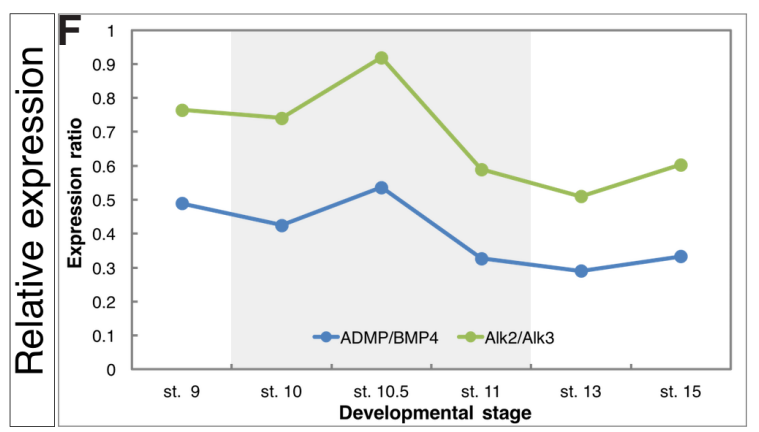

Fig. 2. Temporal expression of the Alk1 receptor family and the main bone morphogenetic protein (BMP ) ligands during gastrulation. $R N A$ samples from $X$. laevis embryos from early cleavage (st. 4-5) to late neurula (st. 22) stages were analyzed by qPCR. Temporal pattern of the receptor gene expression; (A) Alk2 and Alk3, (C) Alk1 and Alk6. Expression of the ligand genes as a function of developmental stage; (B) BMP4, BMP7 and ADMP and (D) BMP2. (E) Relative transcript abundance of the receptor and ligand genes during early/midgastrula (st. 10.5). (F) Comparison of the expression levels between ADMP/ BMP4 and Alk2/Alk3 from late blastula (st. 9) to early neurula (st. 15) stages. The gray shading marks gastrula stages. 
to the ALK1 family of type I of TGF $\beta$ receptors (Yadin et al., 2016). This family of receptors includes ALK1, ALK2, ALK3, and ALK6. In Xenopus embryos, the role of ALK3 and ALK6 as BMP receptors has been the focus of multiple of studies (Graff et al., 1994; Schille et al., 2016). In contrast, the characterization of ALK2 during early embryogenesis lags behind, and very little is known regarding ALK1 (Kondo et al., 1996; Armes and Smith 1997; Chen et al., 1997). To study the pattern of Alk1 and Alk2 expression, we determined their cDNA sequence through data mining of the GenBank and Xenbase databases. The sequence of ALK2 was previously reported (Kondo et al., 1996; Chen et al., 1997), and sequences of the Alk1 cDNAs from Xenopus laevis (ACVRL1.L, XM_018247346; ACVRL1.S, XM_018248624), and Xenopus tropicalis (XM_002935116, XM_012957763) have been predicted (Xenbase.org). We corroborated the sequences of both receptors (Supplementary Fig. S1 A,B). Comparison of all four receptors emphasizes the similarities and differences between the receptors (Fig. 1). Comparison of the Xenopus sequences to the human and mouse receptors identified regions with a high degree of conservation (Fig. 1). We PCR cloned cDNAs of both genes (see Materials and Methods), and designed gene-specific primers for their characterization by quantitative real-time PCR (qPCR).

\section{Temporal expression patterns of the ALK1 family of receptors and their putative ligands}

The temporal expression patterns of $A / k 1$ and $A / k 2$ were determined by qPCR using RNA samples spanning from early cleavage stages (st. 4-5) to advanced neurula stages (st. 22). These temporal expression patterns were compared to the pattern of Alk3 and Alk6 expression using specific primers. Alk2 transcription becomes apparent during late blastula (st. 9; Fig. 2A). Alk2 transcripts continue to accumulate until mid-gastrula (st. 10.5), and then expression levels increase much slower until late gastrula/early neurula (st. 13) when they begin to decline (Fig. 2A). Comparison to $A / k 3$ expression shows that also this gene begins to be transcribed during late blastula, but transcripts of this gene continue to accumulate until the end of gastrulation (Fig. 2A).

Alk1 expression is reminiscent of the expression of $A / k 2$ and Alk3 with the main difference being that $A / k 1$ expression begins with a slight delay such that transcript accumulation begins with the onset of gastrulation (st. 10; Fig. 2C). Using the same RNA samples, we also determined the temporal pattern of Alk6 expression, whose zygotic expression is delayed until mid/late gastrula stages (st. 11; Fig. 2C). These patterns of Alk3 and Alk6 expression are in agreement with previously reported patterns (Graff et al., 1994; Schille et al., 2016).

The temporal expression pattern of the main BMPs during early gastrula, BMP2, BMP4, BMP7, and ADMP (Fainsod et al., 1994; Moos et al., 1995; Hawley et al., 1995; Marom et al., 2005), was also determined using the same RNA samples. These BMPs are the putative ligands of the type I BMP receptors studied. ALK3 and ALK6 have been described as BMP4 receptors (Reversade and de Robertis 2005; Schille et al., 2016). ALK3 also can bind BMP2 and BMP7. ALK2 has been shown to bind ADMP and BMP7. The ligand of ALK1 has remained elusive, and BMP9 has been identified as a ligand of this receptor. $A D M P$ and $B M P 4$ exhibit almost identical temporal expression patterns with zygotic transcripts appearing during late blastula (st. 9) and continue to accumulate until early neurula (st. 13; Fig. 2B). Expression of BMP7 also begins during late blastula (st. 9) and continues to increase until late gastrula (Fig. 2B). Temporal analysis shows that the Xenopus embryo has significant maternal BMP2transcripts. The level of maternal BMP2 transcripts decreases towards the onset of gastrulation, and during mid-, late gastrula (after st. 11) zygotic transcription ensues (Fig. 2D). A similar expression pattern has been previously described for BMP2 (Marom et al., 2005).
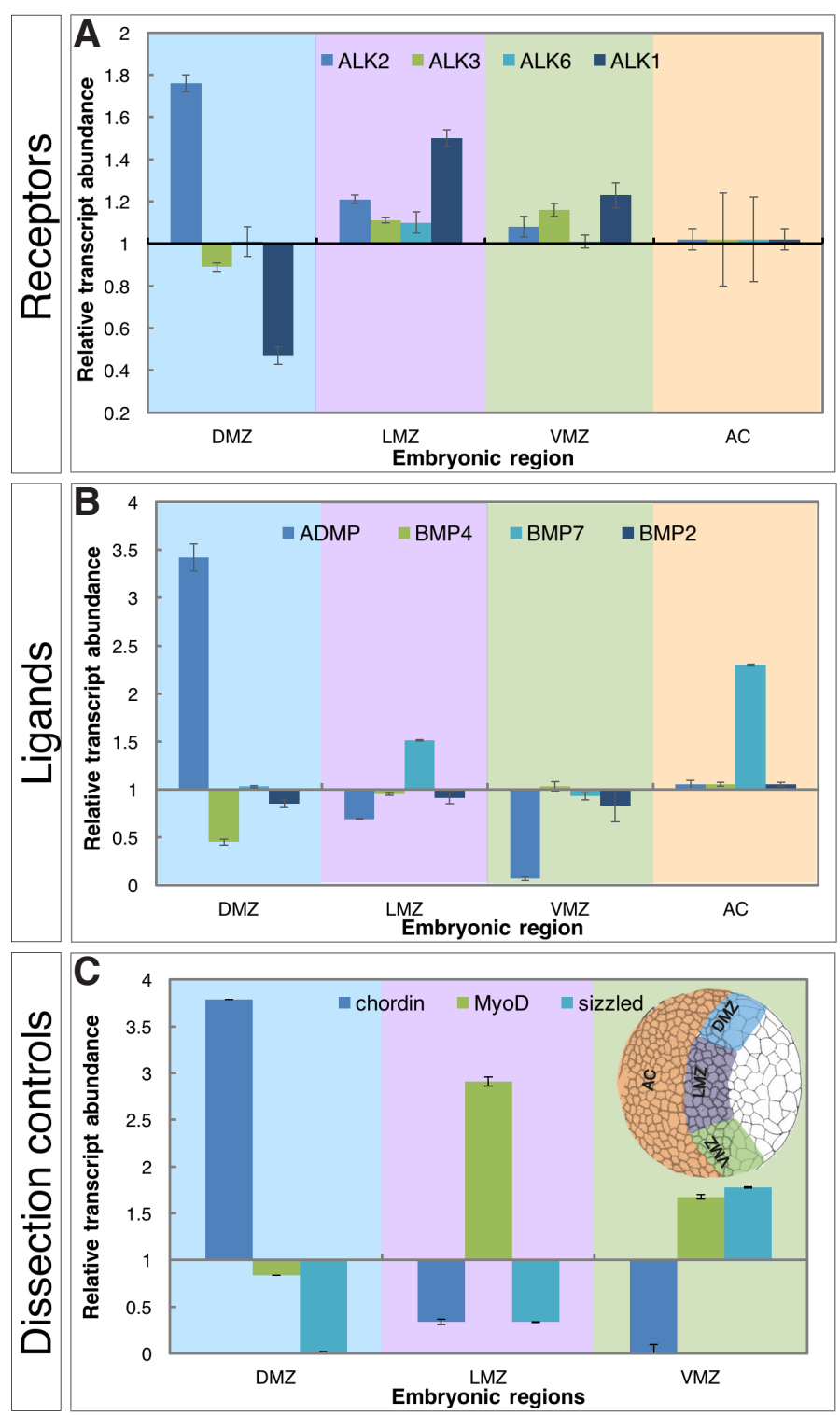

Fig. 3. Spatial localization of the Alk1 family transcripts in gastrula embryos. X. laevis embryos at early/mid-gastrula (st. 10.5) were dissected into four regions, the animal cap (AC), and the dorsal, lateral, and ventral, regions of the marginal zone (DMZ, $L M Z$, and $L M Z$, respectively). RNA from each embryonic region was analyzed by qPCR. Relative transcript abundance in each region was normalized for most genes to the AC level of expression, only for BMP7 the level of expression in the VMZ was used for normalization. (A) Relative transcript abundance of the ALK1 family receptors. (B) Relative spatial localization of the BMP ligand transcripts. (C) The transcript localization of the chordin, MyoD, and sizzled genes was analyzed to determine the dissection accuracy for the $D M Z, L M Z+V M Z$, and $V M Z$ respectively. A schematic representation of the embryo is shown. 
An important observation from the qPCR data was an estimation of the relative expression levels of the different receptors and ligands. Our semi-quantitative comparison revealed that during early/mid-gastrula (st. 10.5) both Alk3 and Alk2 are expressed at very similar levels (Fig. $2 \mathrm{E}$ ). At the same stage, the level of $A D M P$ transcripts is about half the amount of BMP4 transcripts (Fig. 2E). Analysis of the other receptors and ligands revealed that they are expressed at even lower levels (Fig. 2E). The level of Alk1 expression was unusually low in agreement with its temporal delay in expression compared to Alk2 and Alk3 (Figs. 2 C,E). Of particular interest was the comparison between BMP4 and ADMP and their receptors, $A / k 3$ and $A / k 2$, during gastrula stages as they appear to be the most abundant at these stages. Estimation of the relative transcript levels from late blastula (st. 9) to early neurula (st. 15), suggested that $A D M P$ expression declines compared to $B M P 4$, and Alk2 expression becomes less abundant compared to Alk3 (Fig. 2F). Based on the restricted area, the organizer, where $A D M P$ is expressed (Moos et al., 1995), these results suggest that the level of $A D M P$ transcripts there is extremely abundant, and is similar or higher than $B M P 4$ transcripts throughout the rest of the embryo. The high level of $A D M P$ transcripts within the dorsal region again questions its $B M P$-like anti-dorsalizing function.

Comparison of the ALK and BMP temporal expression patterns

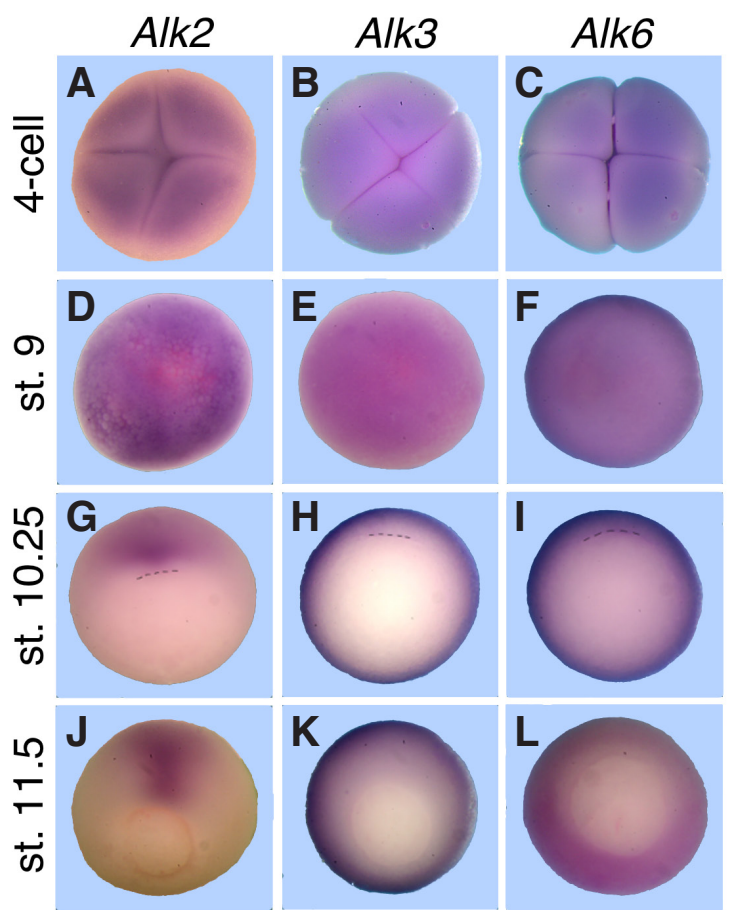

Fig. 4 (left).The Alk2 gene encodes an organizer-expressed gene. Whole-mount ISH analysis of the Alk2, Alk3, and Alk6 genes from early cleavage to late gastrula stages. (A-C) Fourcell embryos, animal view. (D-F) Late blastula stage (st. 9), animal view. (G-I) Early gastrula embryos (st. 10.25), vegetal view. The dorsal lip of the blastopore is marked with a dashed line. (J-L) Late gastrula (st. 11.5) expression, vegetal view.

Fig. 5 (right). Bone morphogenetic protein 7 (BMP7) expression marks the epiboly of the prospective ectoderm. Whole-mount ISH analysis of BMP7 expression from late blastula to early neurula. Lateral views of st. 9 (A), st. 10 (B), st. 10.5 (C), and st. 11 (D) embryos, and posterior animal view of st. 13 (E) embryo. suggests that expression of all four type I receptors studied, partially overlaps in time with BMP4, BMP7, and ADMP. Our temporal analysis did not identify a type I BMP receptor recapitulating the temporal pattern of BMP2 expression, in particular, its high maternal abundance and subsequent decline. The original description of Alk3 reported high maternal transcript levels and a sharp decline during early development (Graff et al., 1994). Analysis of recent high-throughput expression studies (Yanai et al., 2011; Owens et al., 2016) did not corroborate the high Alk3 transcript levels, like our results, but otherwise suggested high maternal Alk2 expression in Xenopus tropicalis. Also, in the high-throughput studies, the transcript levels of Alk1 appear to be very low. Although the temporal analysis suggested possible receptor-ligand interactions, temporal expression overlap also requires spatial co-expression or some proximity.

\section{Spatial localization of the ALK1 and BMP family expression}

To further understand the overlap in the expression patterns of these receptors and ligands, we turned to a comparative analysis of their spatial pattern of expression during gastrulation. To this end, we manually dissected early/mid-gastrula stage embryos (st. 10.25-10.5) into dorsal, lateral and ventral marginal zone regions (DMZ, $L M Z$, and $V M Z$, respectively) and the animal cap

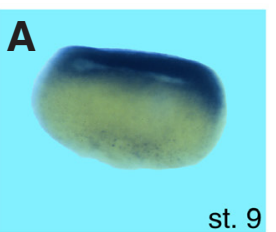

B

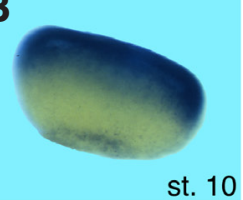

st. 10

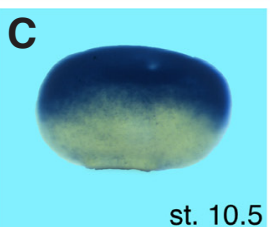

D

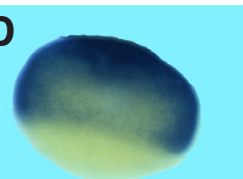

st. 11

st. 13

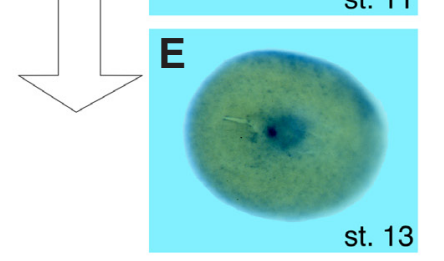
(AC; Fig. 3). Care was taken to remove the animal cap tissue from the $M Z$ explants. RNA was prepared from each region, and qPCR was performed to determine the transcript distribution of the BMP ligands, and the ALK receptors and the relative spatial abundance of the transcripts were estimated (Fig. 3). Among the receptors, most $A / k 2$ transcripts localize to the DMZ, while, Alk1 is mainly expressed in the $L M Z$ with little expression in the VMZ (Fig. 3A). Alk3 and Alk6 expression is widely distributed along the LMZ, and VMZ (Fig. 3A).

Parallel analysis of the BMP ligands revealed high $\mathrm{DMZ}$ abundance of $A D M P$ transcripts (Fig. 3B) as previously described (Moos et al., 1995). BMP4 transcripts can be detected throughout the embryo avoiding only the DMZ (Fig. 3B) in accordance with the described pattern (Fainsod etal., 1994). BMP2 transcripts appear to be widely distributed in all embryonic regions (Fig. 3B) in agreement with its previously published whole-mount in situ hybridization (ISH) pattern (Clement et al., 1995). Transcripts of BMP7appear to be mostly localized to the animal cap region with some accumulation in the $\mathrm{MZ}$, in particular along the LMZ (Fig. 3B) as previously described (Hawley et al., 1995). The qPCR patterns described, corroborate the previously described ISH patterns, and attest to the accuracy of our dissections. Also, we determined the transcript localization of chordin, $M y o D$, and sizzled as markers of the $\mathrm{DMZ}, \mathrm{LMZ}+\mathrm{VMZ}$, and $\mathrm{VMZ}$ respectively (Fig. 3C).

Based on this analysis, both Alk2and ADMP transcripts are predominantly localized to the 


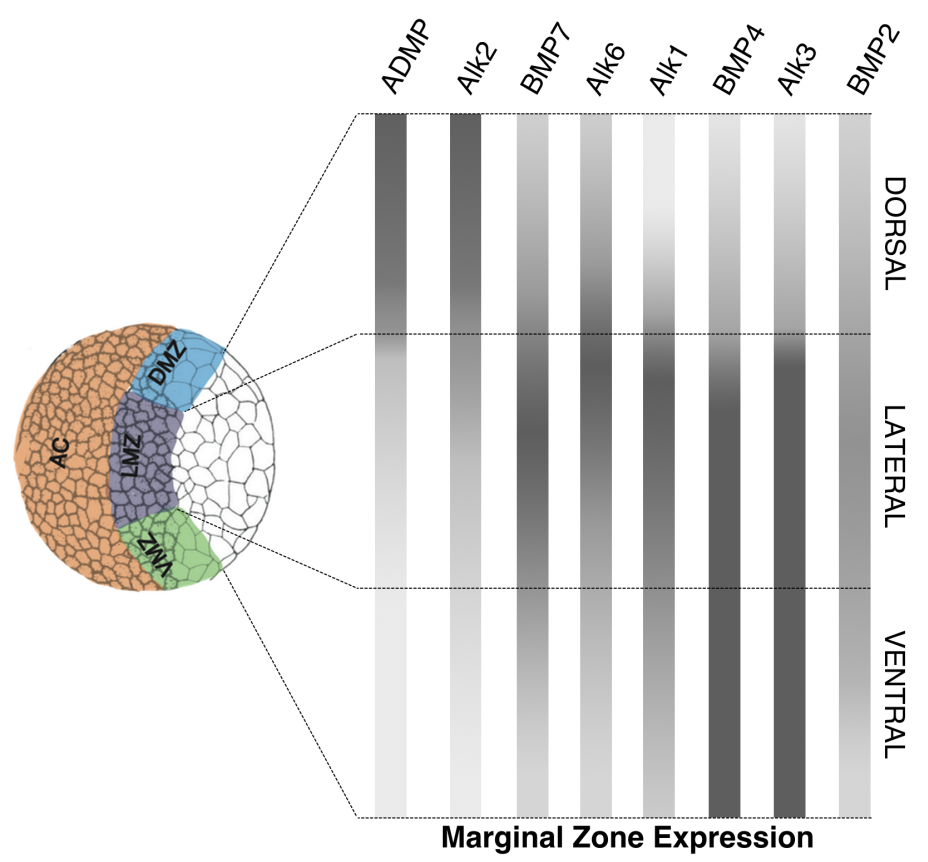

Fig. 6. Expression of the ALK1 receptor family and the bone morphogenetic protein (BMP) ligands during gastrula. Schematic summary of the expression of the type I receptors Alk1, Alk2, Alk3, and Alk6, and the BMPs, BMP2, BMP4, BMP7, and ADMP. The summary focuses on the marginal zone expression of the different genes and relates to the dissection scheme.

DMZ (Figs. 3 A,B) in agreement with ADMP binding to ALK2 (Reversade and de Robertis 2005). To further corroborate the dorsal expression of Alk2 we determined its spatial expression pattern by whole mount ISH. During early cleavage stages (4-cells), some Alk2 transcripts are detected in the animal region, and this localization persists until late blastula stages (st. 9) (Figs. 4 A,D). Expression of Alk2 during early gastrula shows a novel site of expression restricted to the dorsal blastoporal lip, i.e. Spemann's organizer (Fig. 4G). By late gastrula (st. 11.5) the transcripts of Alk2 localize to the dorsal midline of the embryo from the blastopore to rostral regions (Fig. 4J). Our results show that both, ADMP (Moos et al., 1995) and its receptor, ALK2, exhibit extensive expression overlap during gastrula stages, and suggest a possible signaling event mediated by this ligand-receptor pair within the organizer.

For comparison purposes, we also studied the spatial pattern of Alk3 and Alk6 expression. Similarly, both Alk3 and Alk6 transcripts can be observed in the animal region of 4-cell embryos and continue there until late blastula stages (Figs. 4 B,C,E,F). During early (st. 10.25) and late (st. 11.5) gastrula, the animal cap expression persists, and both Alk3 and Alk6 are expressed throughout the marginal zone (Fig. $4 \mathrm{H}, \mathrm{I}, \mathrm{K}, \mathrm{L}$ ). In both cases, during gastrula stages, fewer transcripts appear to be present in the dorsal blastopore region, Spemann's organizer. These spatial patterns of expression together with the temporal analysis show that $A / k 3$ and Alk6 expression extensively overlaps with the BMP4 transcript localization (Fainsod et al., 1994). This overlap further supports the ligand-receptor interaction between BMP4 and its receptors. The extremely low transcript levels of Alk1 precluded the determination of its spatial pattern of expression by whole mount ISH.

The results of $B M P 7$ transcript localization based on embryo fragments placed the expression of this gene mainly to the animal region (Fig. 3B), corroborating the previously published ISH pattern (Hawley et al., 1995). A more detailed analysis of BMP7 expression during gastrula stages revealed a dynamic pattern. From late blastula to late gastrula, the expression of $B M P 7$ localizes mainly to the animal cap cells (Fig. 5 A-D). Interestingly, BMP7 expression persists in the animal cap region as it envelops the embryo. As both, ALK3 and ALK6 are expressed in the animal cap and marginal zone during gastrula stages, they could be mediating some of the BMP7 signaling.

Based on their temporal and spatial synexpression (Fig. 6), we attempted to suggest possible ligand-receptor pairs between the main BMPs and type I receptors during gastrula stages. Based on their expression, ALK3 (BMPR1A) and ALK6 (BMPR1B) are poised to mediate the signaling induced by BMP4 and BMP7 (Fig. $6)$. This suggested ligand-receptor interaction has been identified in some instances (Little and Mullins 2009). The situation for BMP2 is less clear as several ALK1-family receptors seem to have some degree of maternal contribution, but none recapitulates the BMP2 temporal expression pattern. From the expression analysis, the maternal BMP2 transcript level from early cleavage to blastula stages could be equivalent to the level of BMP4 during gastrula stages as we have previously suggested (Marom et al., 2005). Then, relatively low levels of a receptor like ALK3 could mediate its signaling. ALK2 has been shown to mediate some BMP2 signaling, and it is also able to bind ADMP (Reversade and de Robertis 2005). The co-expression of $A D M P$ and $A / k 2$ within Spemann's organizer supports this observation (Fig. 6), but suggests that ADMP might be performing some signaling in this region as ALK2 is known to be a functional, signal-transducing, receptor. The ALK1 receptor appears to be localized to the LMZ from early/late gastrula stages. This expression would place the ALK1 receptor in regions flanking Spemann's organizer. Our analysis of the main BMP ligands didn't identify a factor with a matching expression pattern, suggesting that, the relevant factor remains to be identified. This analysis of the BMP ligands and their putative receptors during gastrula stages revealed a very dynamic and complex signaling milieu observed in different vertebrate species and developmental stages.

\section{Materials and Methods}

\section{Embryo culture and treatments}

Xenopus laevis frogs were purchased from NASCO (Fort Atkinson, $\mathrm{WI})$. Experiments were performed after approval and under the supervision of the Institutional Ethics Committee. Embryos were obtained by in vitro fertilization, incubated in $0.1 \% \mathrm{MBSH}$, and staged according to Nieuwkoop and Faber (1967).

\section{Embryo manipulation and dissection}

Embryos were dissected into four parts: dorsal (DMZ), lateral (LMZ) and ventral marginal zone (VMZ) and animal cap (AC) at stage 10.25, in $1 \% \mathrm{MBSH}$ buffer. Embryonic regions (15- 20 embryos for each region) were then processed for RNA extraction and qPCR.

\section{cDNA clones and constructs}

PCR cloning of $X$. laevis Alk2, Alk6, and Alk1 was performed with the Herculase II Phusion DNA Polymerase (Stratagene) using cDNA samples from stages 10.5-11.

The primers used for cloning were: 
Alk2, 5'AAGGATCCTGTCCTGCGGAATGG3', 5'CAGAATTCCTAACACAGTAATGGGAGAGGC3';

Alk6, 5'GCTTGCCTTTCACATTCTCTTC3', 5'TCCAGTTCCGACAGCTTACAT3';

Alk1, 5'TTCTGTGTCACCTGAAAACCC3', 5'GTCTGCCACTTTCATGCCTTT3'.

Since no sequence for $X$. laevis Alk1 has been published, we took advantage of the predicted mRNA sequences (Xenbase.org) from the genomic data after extensive comparison to the Xenopus tropicalis published data and Alk1 sequences from other vertebrates. All PCR products were verified by sequencing.

\section{RNA extraction and quantitative real-time PCR}

Total RNA from embryos was extracted with the PerfectPure RNA Tissue Kit with DNase (5 Prime, Hamburg, Germany). cDNA was synthesized using the Iscript cDNA Synthesis kit (Bio-Rad Laboratories).

Quantitative real-time PCR ( $\mathrm{qPCR}$ ) was performed using the Bio-Rad CFX384 thermal cycler and LightCycler 48 SYBR Green I Master (Roche). All samples were processed in triplicate. GAPDH expression levels were used for normalization. For each gene, the relative expression was calculated using the $\Delta \Delta$ ct method, as described previously (Livak and Schmittgen 2001). All experiments were repeated at least three times. qPCR primers used:

$\begin{array}{ll}\text { BMP2: } & \text { 5'ACACGGACAGCAGAAAACCA3', } \\ & \text { 5'AACAGCAGCAGGAGCAGAGA3' } \\ \text { BMP4: } & \text { 5'GCAGCCCAGTAAGGATGT3', } \\ & \text { 5'CTTCTGTGCCTGGTAGATTC3' } \\ \text { BMP7: } & \text { 5'TCTCCTTTGGACATACTTCTTGTG3', } \\ & \text { 5'CGCAACCTCCTCTGGATAAA3' } \\ \text { ADMP: } & \text { 5'GCCTTCCGAGCAAGCTTACTT3', } \\ & \text { 5'CCTTGTGGCAACTGTATCTTATTTTA3' } \\ \text { Chordin: } & \text { 5'AACTGCCAGGACTGGATGGT3', } \\ & \text { 5'GGCAGGATTTAGAGTTGCTTC3' } \\ \text { MyoD: } & \text { 5'CCCTGTTTCAATACCTCAGACAT3', } \\ & \text { 5'CGTGCTCATCCTCGTTATGG3' } \\ \text { Sizzled: } & \text { 5'AACAAGGTCTGCTCCTTCCA3', } \\ & \text { 5'CTGTGGGTCTGGTCCGTATC3' } \\ \text { Alk1 (S+L): } & \text { 5'GCTCTGGGGAAACTTGTGTT3', } \\ & \text { 5'CAACGCTCCTTTATGCTGTT3' } \\ \text { Alk2 (S+L): } & \text { 5'TGTTATGGGCAGCAGTGTT3', } \\ & \text { 5'GATGTTCAAGTTACAGAGGTCACT3' } \\ \text { Alk3 (S+L): } & \text { 5'TGGCTCAGGGCTACCATTATT3', } \\ & \text { 5'CACCTTCTCTCCTCTCCATTTC3' } \\ \text { Alk6 (S+L): } & \text { 5'ACAGCAGGAAGGAAGACACA3', } \\ & \text { 5'ACAGTGGTGGTGGCAGTAAC3' }\end{array}$

\section{Whole mount in situ hybridization}

Whole-mount in situ hybridization (ISH) analysis of gene expression was performed as described previously (Marom et al., 2005). Digoxigeninlabeled RNA probes were prepared from linearized plasmids transcribed in vitro using the RiboMax kit (Promega) and the digoxigenin RNA labeling mixture (Roche). Probes for ISH were transcribed from pSP64TNE BMPR clone for Alk3.L (Graff et al., 1994). DNA templates utilized for transcription of Alk2 and Alk6 in situ hybridization probes were generated using PCR amplification. The primers used: ALK2_DIG (S+L), 5'TGACCTCTGTAACTTGAACATCAC3', 5'ATTGCTGACCATCCGTCTG3';ALK6_DIG (S+L), 5'CGTTTCCTTGATTATGTTGCTATC3', 5'TATTTCAGTGTGTAGGTGGCAGA3'. The T7 promoter sequence (TAATACGACTCACTATAGGG) was added to the reverse primer for transcription.

\section{Acknowledgements}

This paper is dedicated to the memory of Herbert Steinbeißer, who passed away in 2014. He was involved in this project from its beginning and the BMP7 analysis was performed by him. This project was supported in part by grants from United-States Binational Science Foundation (No. 2013422), Israel Cancer Research Fund (NA), the Chief Scientist of the Israel Ministry of Health (No. 3-0000-10068) and the Wolfson Family Chair in Genetics to AF.

\section{References}

ARMES N A, SMITH J C (1997). The ALK-2 and ALK-4 activin receptors transduce distinct mesoderm-inducing signals during early Xenopus development but do not co-operate to establish thresholds. Development 124: 3797-3804.

CHEN Y, BHUSHAN A, VALE W (1997). Smad8 mediates the signaling of the ALK-2 [corrected] receptor serine kinase. Proc Natl Acad Sci USA 94: 12938-12943.

CLEMENT JH, FETTESP, KNÖCHELS, LEFJ, KNÖCHELW (1995). Bone morphogenetic protein 2 in the early development of Xenopus laevis. Mech Dev52:357-370.

DOSCH R, GAWANTKA V, DELIUS H, BLUMENSTOCK C, NIEHRS C (1997). Bmp-4 acts as a morphogen in dorsoventral mesoderm patterning in Xenopus. Development 124: 2325-2334.

FAINSODA, STEINBEISSER H, DE ROBERTISEM (1994). On the function of BMP-4 in patterning the marginal zone of the Xenopus embryo. EMBO J 13: 5015-5025.

GRAFF J M, THIES R S, SONG J J, CELESTE A J, MELTON D A (1994). Studies with a Xenopus BMP receptor suggest that ventral mesoderm-inducing signals override dorsal signals in vivo. Cell 79: 169-179.

HAWLEY S H, WÜNNENBERG-STAPLETON K, HASHIMOTO C, LAURENT M N, WATABE T, BLUMBERG B W, CHO K W (1995). Disruption of BMP signals in embryonic Xenopus ectoderm leads to direct neural induction. Genes Dev 9: 2923-2935.

HELDIN C-H, MOUSTAKASA (2016). Signaling Receptors for TGF- $\beta$ Family Members. Cold Spring Harb Perspect Biol 8: a022053.

KONDO M, SEMBA K, SHIOKAWA K, YAMAMOTO T (1996). Molecular cloning of Xenopus activin type I receptor and the analysis of its expression during embryogenesis. Biochem Biophys Res Commun 218: 549-555.

LITTLE S C, MULLINS M C (2009). Bone morphogenetic protein heterodimers assemble heteromeric type I receptor complexes to pattern the dorsoventral axis. Nat Cell Biol 11:637-643. doi: 10.1038/ncb1870

LIVAK K J, SCHMITTGEN T D (2001). Analysis of relative gene expression data using real-time quantitative PCR and the 2(-Delta Delta $\mathrm{C}(\mathrm{T})$ ) Method. Methods 25: 402-408.

MAROM K, FAINSOD A, STEINBEISSER H (1999). Patterning of the mesoderm involves several threshold responses to BMP-4 and Xwnt-8. Mech Dev87:33-44.

MAROM K, LEVY V, PILLEMER G, FAINSOD A (2005). Temporal analysis of the early BMP functions identifies distinct anti-organizer and mesoderm patterning phases. Dev Biol 282: 442-454.

MOOS M, WANG S, KRINKS M (1995). Anti-dorsalizing morphogenetic protein is a novel TGF-beta homolog expressed in the Spemann organizer. Development 121: 4293-4301.

NIEUWKOOP P D, FABER J (1967). Normal table of Xenopus laevis (Daudin): A systematical and chronological survey of the development from the fertilized egg till the end of metamorphosis., 2nd edn. North-Holland Publishing Company, Amsterdam.

OWENS N D L, BLITZ I L, LANE M A, PATRUSHEV I, OVERTON J D, GILCHRIST M J, CHO K W Y, KHOKHA M K (2016). Measuring Absolute RNA Copy Numbers at High Temporal Resolution Reveals Transcriptome Kinetics in Development Cell Rep 14: 632-647.

REVERSADE B, DE ROBERTIS E M (2005). Regulation of ADMP and BMP2/4/7 at opposite embryonic poles generates a self-regulating morphogenetic field. Cell 123: 1147-1160.

SCHILLE C, HELLER J, SCHAMBONY A (2016). Differential requirement of bone morphogenetic protein receptors la (ALK3) and lb (ALK6) in early embryonic patterning and neural crest development. BMC Dev Biol 16: 1.

YADIN D, KNAUS P, MUELLER T D (2016). Structural insights into BMP receptors: Specificity, activation and inhibition. Cytokine Growth Factor Rev 27: 13-34.

YANAI I, PESHKIN L, JORGENSEN P, KIRSCHNER M W (2011). Mapping gene expression in two Xenopus species: evolutionary constraints and developmental flexibility. Dev Cell 20: 483-496. 


\section{Further Related Reading, published previously in the Int. J. Dev. Biol.}

Functional differences between Tcf1 isoforms in early Xenopus development

Giulietta Roël, Olaf Van Den Broek and Olivier Destrée

Int. J. Dev. Biol. (2017) 61: 29-34

https://doi.org/10.1387/ijdb.160308od

Epithelial cell division in the Xenopus laevis embryo during gastrulation

Guillaume Hatte, Marc Tramier, Claude Prigent and Jean-Pierre Tassan

Int. J. Dev. Biol. (2014) 58: 775-781

https://doi.org/10.1387/ijdb.140277jt

Expression of xSDF-1a, xCXCR4, and XCXCR7 during gastrulation in Xenopus laevis Surabhi-Kirti Mishra, Tomoko Nagata, Kazuya Furusawa,Naoki Sasaki and Akimasa Fukui Int. J. Dev. Biol. (2013) 57: 95-100

https://doi.org/10.1387/ijdb.120130af

The Wnt signaling mediator tcf1 is required for expression of foxd3 during Xenopus gastrulation

Sylvie Janssens, Olaf Van Den Broek, lan R. Davenport, Robbert C. Akkers, Fei Liu, Gert Jan C. Veenstra, Stefan Hoppler, Kris Vleminckx and Olivier Destrée

Int. J. Dev. Biol. (2013) 57: 49-54

https://doi.org/10.1387/ijdb.120191kv

Xler2 is required for convergent extension movements during Xenopus development Sung-Kook Hong, Kosuke Tanegashima and Igor B. Dawid

Int. J. Dev. Biol. (2011) 55: 917-921

https://doi.org/10.1387/ijdb.113288sh

Retinoid signalling is required for information transfer from mesoderm to neuroectoderm during gastrulation

Ferran Lloret-Vilaspasa, Hans J. Jansen, Koen de Roos, Rosh A.S. Chandraratna, Maija H. Zile, Claudio D. Stern and Antony J. Durston

Int. J. Dev. Biol. (2010) 54: 599-608

https://doi.org/10.1387/ijdb.082705fl

Xenopus development from late gastrulation to feeding tadpole in simulated microgravity Wendy M. Olson, Darrell J. Wiens, Theresa L. Gaul, Manuela Rodriguez and Chérie L. Hauptmeier

Int. J. Dev. Biol. (2010) 54: 167-174

https://doi.org/10.1387/ijdb.072562wo

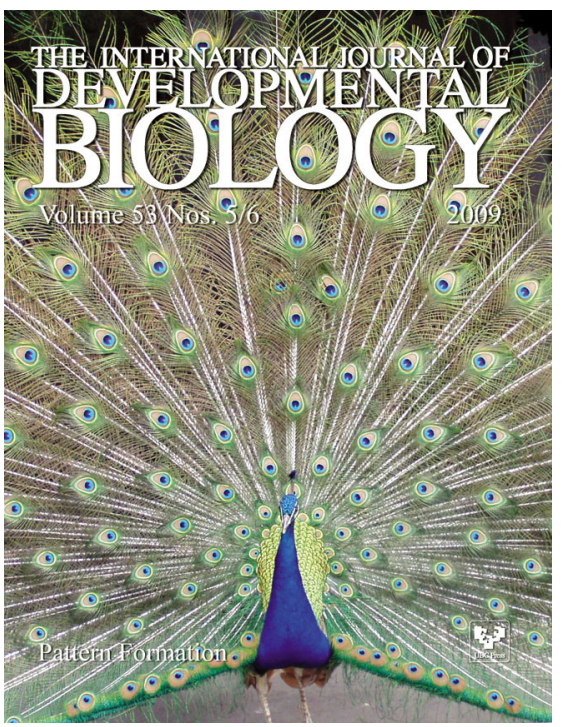

5 yr ISI Impact Factor $(2013)=2.879$
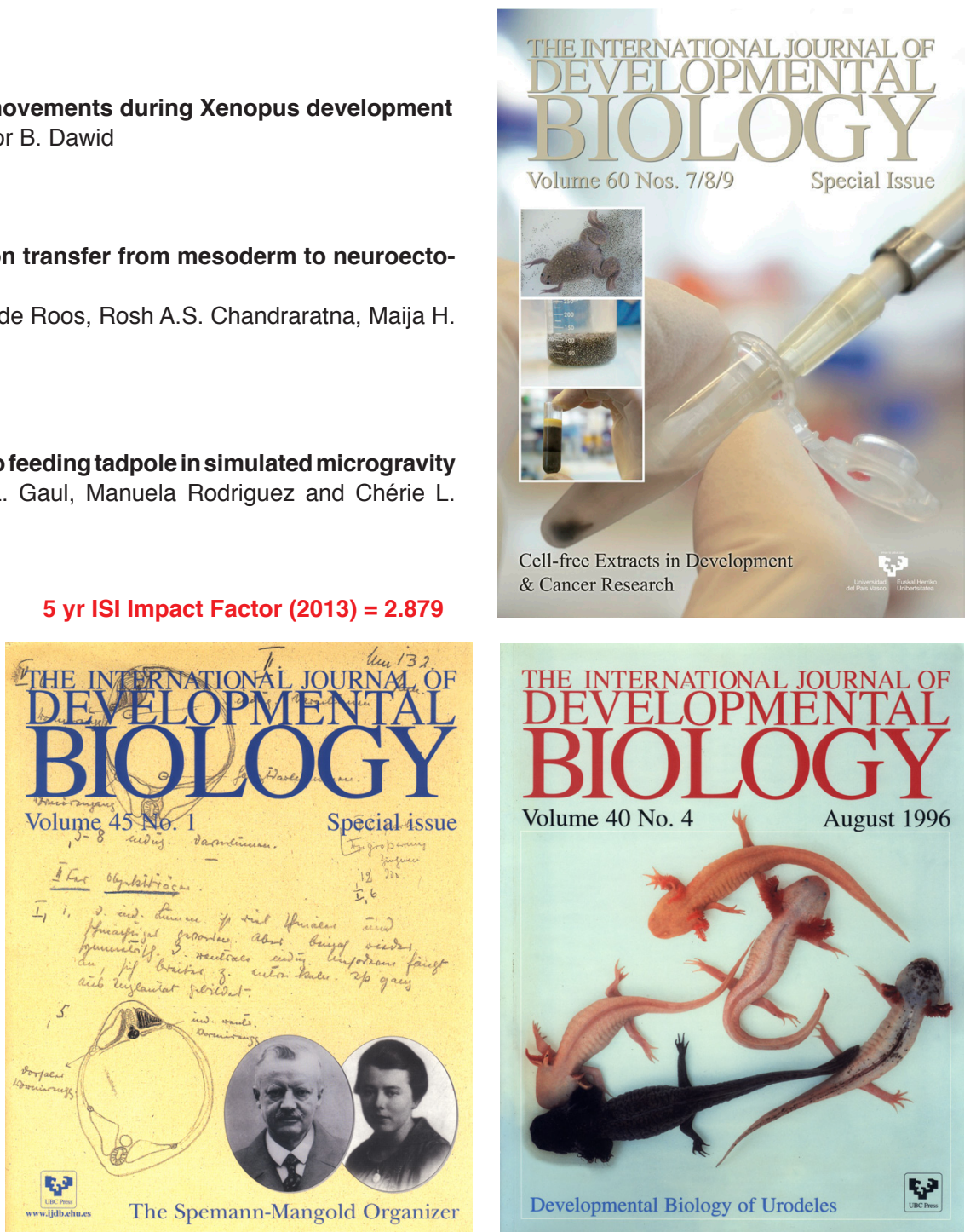

Developmental Biology of Urodeles 